\title{
The International Council for Harmonisation and a Case Study in Regulatory Science
}

\author{
Therapeutic Innovation \\ \& Regulatory Science \\ 2019, Vol. 53(5) 561-563 \\ (C) DIA 2019 \\ Article reuse guidelines: \\ sagepub.com/journals-permissions \\ DOI: I0.II77/2I684790I9869740 \\ tirs.sagepub.com
}

Regulatory science is a fundamental component of contemporary new drug development, and discussions of its role can be found in publications in this and other journals. ${ }^{1,2}$ While many organizations and consortia are involved in this science, one of particular note is the International Council for Harmonisation of Technical Requirements of Pharmaceuticals for Human Use (ICH). ${ }^{3} \mathrm{ICH}$ represents an amalgamation of clinical, scientific, and regulatory expertise from academic institutions, pharmaceutical organizations, and regulatory agencies across the world. It arose because the regulations for submitting documentation requesting marketing approval of a drug were historically quite different between countries. Data requirements were dissimilar, meaning that more studies often had to be conducted to satisfy national regulatory requirements if marketing permission was desired in multiple countries. This lack of uniformity meant that animal (nonclinical) and human (clinical) studies may have had to be repeated in slightly different ways, resulting in additional and unnecessary use of animal, human, and financial resources. Consequently, it meant that bringing a drug to market in various countries took longer than necessary, thereby delaying its availability to patients.

Harmonization of regulatory requirements was pioneered by the European Community (now the European Union) in the $1980 \mathrm{~s}$ as it moved towards the development of a single market for pharmaceuticals. The success achieved in Europe demonstrated that harmonization was feasible. The process was then extended to include Japan and the United States, and ICH was formed in 1990 by the inclusion of a government body and an industry association from each of these regions. Since then, ICH has evolved to respond to the increasingly global nature of new drug development. As one indicator of its growth, almost 500 participants from its 16 Member and 32 Observer organizations (the latter including the World Health Organization and the Bill \& Melinda Gates Foundation) attended its biannual meeting in June 2019. ${ }^{4}$

As noted on its web site, ${ }^{3}$ ICH's mission is "to achieve greater harmonisation worldwide to ensure that safe, effective, and high-quality medicines are developed and registered in the most resource-efficient manner." Goals to enable this include the following:

- Maintain a forum for constructive dialog between regulatory authorities and the pharmaceutical industry on differences in technical requirements for marketing approval to ensure more timely introduction of new drugs to the market.

- Facilitate the adoption of new or improved technical research and development approaches that update or replace current practices. These new or improved practices should permit a more economical use of animal, human, and material resources without compromising safety (for discussion of the $3 \mathrm{R}$ principles for reducing animal investigation, see Törnqvist et $\mathrm{al}^{5}{ }^{5}$ Aske and Waugh, ${ }^{6}$ and Franco et $\mathrm{al}^{7}$ ).

- Monitor and update harmonized technical requirements, leading to a greater mutual acceptance of research and development data.

- Contribute to the protection and promotion of public health from an international perspective.

- Encourage the implementation and integration of common standards of documentation and submission of regulatory applications by disseminating harmonized guidelines.

To facilitate the last goal, the ICH has produced many guidelines for sponsors to use in various aspects of drug development research and documentation: these guidelines are typically adopted by regulatory agencies on their release. Guidelines are grouped into 4 categories - quality, safety, efficacy, and multidisciplinary. The genesis, release, and periodic revision of 2 of these guidelines, ICH S7B and ICH E14, are discussed in this Editorial as a case study in regulatory science.

\section{ICH S7B, ICH EI4, and the Evolution of the Science of Proarrhythmic Cardiac Safety}

For reasons of cardiac physiology that need not be discussed here (for details, see Satin et $\mathrm{al}^{8}$ and Turner et $\mathrm{al}^{9,10}$ ), a wide range of drugs for many conditions can predispose individuals with certain clinical and demographic characteristics to experience cardiac arrhythmias by affecting the heart's electrical activity. Of particular interest is drug-induced torsade de pointes (torsade), a rare polymorphic ventricular arrhythmia typically occurring in self-limiting bursts that can lead to symptoms of dizziness, palpitations, syncope, and seizures. Additional clinical concern arises because this arrhythmia also has potentially catastrophic consequences - it can occasionally progress to ventricular fibrillation and sudden cardiac death. ${ }^{11}$ Precipitating risk factors include advanced age, female sex, 
structural heart disease, metabolic and electrolyte abnormalities including hypokalemia and hypocalcemia, bradycardia and cardiac conduction disease, increased drug bioavailability due to renal or hepatic impairment, genetic predisposition (a clinical condition called long QT syndrome, which in itself is associated with torsade), and the co-prescription of loop diuretics and other proarrhythmic drugs. ${ }^{12-14}$ As Link and colleagues observed, "One of the most feared complications in medicine is sudden death caused by drug-induced proarrhythmia. Accordingly, concerted efforts have been made to define a drug's proarrhythmic potential before regulatory approval." ${ }^{\text {15 }}$

These efforts have their origins in the release by $\mathrm{ICH}$ in 2005 of 2 guidelines, ICH S7B, ${ }^{16}$ which guides nonclinical investigations, and ICH E14, ${ }^{17}$ which guides clinical assessments. These documents were subsequently adopted by multiple regulatory agencies. ICH E14 focused on one particular aspect of the electrocardiogram (ECG), the QT interval. We will discuss ICH S7B in due course, first focusing on ICH E14.

A dedicated clinical pharmacology study introduced by ICH E14, the Thorough QT Study, determines whether or not administration of a new drug leads to notable lengthening (prolongation) of the QT interval. Drugs that are deemed to have a considerable effect on the QT interval have had warning language involving QT interval prolongation placed in their Prescribing Information when approved for marketing: prescribing physicians are thereby alerted to use the drug judiciously by taking into account each patient's clinical risk profile. The approach put forth in this guideline (the same is true for ICH S7B) was based on the best science available at the time. As would be hoped, the science of proarrhythmic cardiac safety has advanced over time. Accordingly, an ICH E14 "Questions \& Answers" document was released in June 2008, and revisions to this document were released in April 2012, March 2014, and December 2015, ${ }^{18}$ respectively. With each revision, additional questions were asked and answered, providing drug developers with discussions of the latest scientific activities and new potential pathways for assuring regulatory agencies that a new drug under development does not carry an unacceptable proarrhythmic liability.

Of interest to many of us in the field of proarrhythmic safety will be the outcome of discussions concerning another potential ICH document during the aforementioned ICH meeting 3 months ago. Many guideline-related Working Groups met together, and of interest here is the meeting of the ICH E14/ S7B Implementation Working Group (IWG). While the contents of their discussions are confidential at this time, several documents on ICH's web site provide a publicly available architectural framework portending the general nature of these discussions. A finalized Concept Paper entitled "ICH S7B and E14 Q\&A" that was released in November 2018 outlines the issues at hand, ${ }^{19}$ an IWG Work Plan prepared in February 2019 details the current status of key milestones and the nature and timing of future anticipated key milestones, ${ }^{20}$ and an E14/S7B IWG Membership List provides participants' names and their affiliations: members come from pharmaceutical organizations and regulatory agencies in Canada, China, Europe, Japan, Switzerland, Turkey, and the United States. ${ }^{21}$

As noted previously, to date there have been 4 updates on clinical assessments via the release of the ICH E14 "Questions \& Answers" document and its 3 revisions. Given ICH's recent work, formalized updates on nonclinical investigations are now likely to be forthcoming. ICH S7B largely focuses on 2 activities: (1) assessment of any drug-induced influence on one specific aspect of the heart's electrophysiological activity, a cardiac potassium ionic current that influences the process of repolarization (the heart getting ready to beat again following the previous beat); and (2) assessment of any drug-induced change in QT interval duration in the ECGs of various animal models, including larger mammalian species. Since 2005, expertise in and experience with more sophisticated in vitro and in silico (computer-based) methodologies has advanced considerably. These advances are represented in the Comprehensive In Vitro Proarrhythmia (CiPA) Initiative.

The CIPA Initiative ${ }^{22-25}$ is driven by an international consortium comprising multiple collaborators and administered by the Health and Environmental Sciences Institute. The first of 3 nonclinical components of CiPA investigates drug-induced effects on multiple isolated human depolarizing and repolarizing ionic currents of interest, including potassium, calcium, and sodium currents: it has become widely accepted that, in addition to the potassium current discussed in S7B in 2005, drug-induced effects on multiple other currents need to be considered. The second component is the use of in silico models of cellular human cardiac (specifically ventricular) activity to integrate drug effects on multiple cardiac currents mathematically, hence providing reconstructions of overall cellular electrical activity. Third, drug-induced effects on the electrical activity of human induced pluripotent stem cell-derived cardiomyocytes are evaluated, providing a representation of a cell-based integrated electrophysiological drug response. Finally, drug-induced effects on human ECGs collected in early clinical pharmacology studies will be evaluated for unanticipated electrophysiologic effects. ${ }^{26}$

If all proceeds smoothly, more information about the contents of the ICH S7B/E14 Q\&A document will become available around June 2020. Society in general (eg, think milestone birthdays and wedding anniversaries) seems to have a fondness for numbers ending in a zero or a five, and, if achieved, this date would register on both scales-2020 ends with a zero and it would also be the 15th anniversary of the 2005 release of ICH S7B and ICH E14. Whatever the exact date, this case study has provided an example of the work of ICH in the domain of regulatory science.

\section{J. Rick Turner, PhD, DSc Editor Emeritus, TIRS}

\section{References}

1. Rouse R, Kruhlak N, Weaver J, et al. Translating new science into the drug review process: the US FDA's Division of Applied 
Regulatory Science. Therapeutic Innovation \& Regulatory Science. 2018;52:244-255.

2. Tan-Koi WC, Leow PC, Teo YY. Applications of pharmacogenomics in regulatory science: a product life cycle review. Pharmacogenomics J. 2018;18:359-366.

3. International Council for Harmonisation of Technical Requirements of Pharmaceuticals for Human Use. www.ich.org (accessed July 7, 2019).

4. ICH press release. file://C:/Users/owner/AppData/Local/Micro soft/Windows/INetCache/IE/JDC9IEBW/ICH38Amsterdam_ PressRelease_2019_0614_Final.pdf (accessed July 7, 2019).

5. Törnqvist E, Annas A, Granath B, et al. Strategic focus on $3 \mathrm{R}$ principles reveals major reductions in the use of animals in pharmaceutical toxicity testing. PLoS One. 2014;9:e101638.

6. Aske $\mathrm{KC}$, Waugh $\mathrm{CA}$. Expanding the $3 \mathrm{R}$ principles: more rigour and transparency in research using animals. EMBO Rep. 2017;18: 1490-1492.

7. Franco NH, Sandøe P, Olsson IAS. Researchers' attitudes to the 3Rs - an upturned hierarchy? PLoS One. 2018;13(8):e0200895.

8. Satin LZ, Durham TA, Turner JR. Assessing a drug's proarrhythmic liability: an overview of computer simulation modeling, nonclinical assays, and the Thorough QT/QTc Study. Drug Info J. 2011:45;357-375.

9. Turner JR, Karnad DR, Cabell CH, Kothari S. Recent developments in the science of proarrhythmic cardiac safety of new drugs. Eur Heart J Cardiovasc Pharmacother. 2017;3:118-124.

10. Turner JR, Rodriquez I, Mantovani E, et al; on behalf of the Cardiac Safety Research Consortium. Drug-induced proarrhythmia and torsade de pointes: a primer for students and practitioners of medicine and pharmacy. J Clin Pharmacol. 2018; 58:997-1012.

11. Vicente J, Strauss DG, Upreti VV, et al. The potential role of the J-Tpeak interval in proarrhythmic cardiac safety: current state of the science from the American College of Clinical Pharmacology and the Cardiac Safety Research Consortium. J Clin Pharmacol. 2019;59:909-914.

12. Vlachos K, Georgopoulos S, Efremidis M, Sideris A, Letsas KP. An update on risk factors for drug-induced arrhythmias. Expert Rev Clin Pharmacol. 2016;9:117-127.

13. Vandael E, Vandenberk B, Vandenberghe J, Willems R, Foulon V. Risk factors for QTc-prolongation: systematic review of the evidence. Int J Clin Pharm. 2017;39:16-25.

14. Heemskerk CPM, Pereboom M, van Stralen K, et al. Risk factors for QTc interval prolongation. Eur J Clin Pharmacol. 2018;74: 183-191.
15. Link MG, Yan GX, Kowey PR. Evaluation of toxicity for heart failure therapeutics: studying effects on the QT interval. Circ Heart Fail. 2010;3:547-555.

16. ICH S7B. The non-clinical evaluation of the potential for delayed ventricular repolarization (QT interval prolongation) by human pharmaceuticals. file://C:/Users/owner/AppData/Local/Micro soft/Windows/INetCache/IE/JDC9IEBW/S7B_Guideline.pdf (accessed July 7, 2019).

17. ICH E14. The clinical evaluation of QT/QTc interval prolongation and proarrhythmic potential for nonantiarrhythmic drugs. file://C:/Users/owner/AppData/Local/Microsoft/Windows/INet Cache/IE/2VUBCJEZ/E14_Guideline.pdf (accessed July 7, 2019).

18. E14 Implementation Working Group ICH E14 Guideline: The clinical evaluation of QT/QTc interval prolongation and proarrhythmic potential for non-antiarrhythmic drugs: questions \& answers (R3). file:///C:/Users/owner/AppData/Local/Microsoft/ Windows/INetCache/IE/0GZPBQG6/E14_Q_As_R3_Step4.pdf (accessed July 7, 2019).

19. ICH. Concept paper. file:///C:/Users/owner/AppData/Local/ Microsoft/Windows/INetCache/IE/JDC9IEBW/E14S7BIWG_ ConceptPaper_Final_2018_1122.pdf (accessed July 7, 2019).

20. ICH E14/S7B IWG work plan. file://C:/Users/owner/AppData/ Local/Microsoft/Windows/INetCache/IE/QILS3H1V/E14-S7B_ IWG_WorkPlan_2019_0220.pdf (accessed July 7, 2019).

21. ICH E14/S7B IWG membership list. file:///C:/Users/owner/App Data/Local/Microsoft/Windows/INetCache/IE/2VUBCJEZ/E14S7B_IWG_ExpertsList_2019_0208.pdf (accessed July 7, 2019).

22. Sager PT, Gintant G, Turner JR, Pettit S, Stockbridge N. Rechanneling the cardiac proarrhythmia safety paradigm: a meeting report from the Cardiac Safety Research Consortium. Am Heart J. 2014;167:292-300.

23. Gintant G, Sager PT, Stockbridge N. Evolution of strategies to improve preclinical cardiac safety testing. Nat Rev Drug Discov. 2016;15:457-471.

24. Vicente J, Zusterzeel R, Johannesen L, et al. Mechanistic modelinformed proarrhythmic risk assessment of drugs: review of the "CiPA" Initiative and design of a prospective clinical validation study. Clin Pharmacol Ther. 2018;103:54-66.

25. Wallis R, Benson C, Darpo B, et al. CiPA challenges and opportunities from a non-clinical, clinical and regulatory perspectives. An overview of the safety pharmacology scientific discussion. J Pharmacol Toxicol Methods. 2018;93:15-25.

26. Vicente J. Update on the ECG component of the CiPA initiative. J Electrocardiol. 2018;51(6 suppl): S98-S102. 